International Journal of Pure and Applied Mathematics

Volume 106 No. 2 2016, 463-471

ISSN: 1311-8080 (printed version); ISSN: 1314-3395 (on-line version)

url: http://www.ijpam.eu

doi: 10.12732/ijpam.v106i2.10

\title{
1-MOVABLE CLIQUE DOMINATING SETS OF A GRAPH
}

\author{
Teffany V. Daniel ${ }^{1}$, Sergio R. Canoy, Jr. ${ }^{2} \S$ \\ ${ }^{1}$ Department of Mathematics \\ Bukidnon State University \\ Malaybalay City, PHILIPPINES \\ ${ }^{2}$ Department of Mathematics and Statistics \\ Mindanao State University-Iligan Institute of Technology \\ Andres Bonifacio Avenue, Tibanga, Iligan City 9200, PHILIPPINES
}

\begin{abstract}
A clique (convex) dominating set $S$ of $G$ is a 1-movable clique dominating set (resp. 1-movable convex dominating set) of $G$ if for every $v \in S$, either $S \backslash\{v\}$ is a clique (resp. convex) dominating set or there exists a vertex $u \in(V(G) \backslash S) \cap N_{G}(v)$ such that $(S \backslash\{v\}) \cup\{u\}$ is a clique (resp. convex) dominating set of $G$. The minimum cardinality of a 1-movable clique (resp. 1-movable convex) dominating set of $G$, denoted by $\gamma_{m c l}^{1}(G)$ (resp. $\gamma_{m c o n}^{1}(G)$ ), is called the 1-movable clique domination number (resp. 1-movable convex domination number) of $G$. A 1-movable clique dominating set in $G$ with cardinality $\gamma_{m c l}^{1}(G)$ is called a $\gamma_{m c l}^{1}$-set of $G$.

This paper aims to characterize the 1-movable clique dominating sets of some graphs including those resulting from the join and composition of two graphs. The corresponding 1-movable clique domination number of the resulting graph is then determined. Further, it is shown that the concepts of 1-movable clique domination and 1-movable convex domination are equivalent.
\end{abstract}

AMS Subject Classification: 05C69

Key Words: clique domination, convex domination, 1-movable clique domination, 1movable convex domination

\section{Introduction}

Let $G=(V(G), E(G))$ be a graph with $n=|V(G)|$ and $m=|E(G)|$. For any vertex $v \in V(G)$, we define the open neighborhood of $v$ as the set $N_{G}(v)=$

Received: September 2, 2015

Published: February 15, 2016

$\S$ Correspondence author
(C) 2016 Academic Publications, Ltd. url: www.acadpubl.eu 
$\{u \in V(G): u v \in E(G)\}$ and the closed neighborhood of $v$ as the set $N_{G}[v]=$ $N_{G}(v) \cup\{v\}$. If $S$ is a nonempty subset of $X$, then $N_{G}(S)=\bigcup_{v \in S} N_{G}(v)$ and $N_{G}[S]=N_{G}(S) \cup S$. A nonempty subset $S$ of $V(G)$ is a dominating set of $G$ if for every $v \in V(G) \backslash S$, there exists $u \in S$ such that $u v \in E(G)$, that is $N_{G}[S]=V(G)$. The domination number of $G$, denoted by $\gamma(G)$, is the minimum cardinality among all dominating sets of $G$. A dominating set $S$ of $G$ with $|S|=\gamma(G)$ is called a $\gamma$-set of $G$. Domination in graph as well as its variants and the numerous applications of these related concepts in networks have been widely studied. The book by Haynes et al. (see [6]) contains a long list of some variations of the standard domination concept.

Now, given two vertices $u$ and $v$ of $G$, by $I_{G}[u, v]$ we mean the closed interval consisting of $u, v$ and all vertices lying on some $u-v$ geodesic (shortest path connecting $u$ and $v$ ) of $G$. A subset $C$ of $V(G)$ is convex if $I_{G}(u, v) \subseteq C$ for every pair of vertices $u, v \in C$. A proper convex subset of $V(G)$ of largest cardinality is called a maximum convex set in $G$. The cardinality of a maximum convex set in $G$ is called the convexity number of $G$ and is denoted by $\operatorname{con}(G)$. A dominating set $S$ of $V(G)$ is a convex dominating set of $G$ if $S$ is convex.

A dominating set $S$ of $V(G)$ is a clique dominating set of $G$ if the induced subgraph $\langle S\rangle$ of $S$ is complete. A clique (convex) dominating set $S$ of $G$ is a 1-movable clique dominating set (resp. 1-movable convex dominating set) of $G$ if for every $v \in S$, either $S \backslash\{v\}$ is a clique (resp. convex) dominating set or there exists a vertex $u \in(V(G) \backslash S) \cap N_{G}(v)$ such that $(S \backslash\{v\}) \cup\{u\}$ is a clique (resp. convex) dominating set of $G$. The minimum cardinality of a 1-movable clique (resp. 1-movable convex) dominating set of $G$, denoted by $\gamma_{m c l}^{1}(G)$ (resp. $\gamma_{m c o n}^{1}(G)$ ), is called the 1-movable clique domination number (resp. 1-movable convex domination number) of $G$. A 1-movable clique dominating set in $G$ with cardinality $\gamma_{m c l}^{1}(G)$ is called a $\gamma_{m c l}^{1}$-set of $G$.

The concept of clique domination is studied by Cozzens and Kelleher in [4]. Convexity and some of its related concepts are studied and investigated in [2], [3], and [5] while convex domination in graphs are dealt with in [8] and [9]. Blair et al. introduced and studied movable domination in [1]. The concept of 1-movable domination is also studied in [7].

This paper investigates, among others, the concept of 1-movable clique domination in the join and composition of graphs. Recall that the join of two graphs $G$ and $H$ is the graph $G+H$ with vertex set $V(G+H)=V(G) \cup V(H)$ and edge set $E(G+H)=E(G) \cup E(H) \cup\{u v: u \in V(G), u \in V(H)\}$. The composition (sometimes called lexicographic product) of $G$ and $H$ is the graph $G[H]$ with $V(G[H])=V(G) \times V(H)$ and $(u, v)\left(u^{\prime}, v^{\prime}\right) \in E(G[H])$ if and only if either 
$u u^{\prime} \in E(G)$ or $u=u^{\prime}$ and $v v^{\prime} \in E(H)$.

\section{Results}

The first result shows that the concepts of 1-movable clique domination and 1-movable convex domination are equivalent.

Theorem 2.1. Let $G$ be a connected graph. Then $G$ has a 1-movable convex dominating set if and only if it has a 1-movable clique dominating set. Moreover, $S$ is a 1-movable convex dominating set of $G$ if and only if it is a 1-movable clique dominating set.

Proof. Suppose that $S$ is a 1-movable convex dominating set of $G$. Suppose further that $\langle S\rangle$ is not complete. Then there exist vertices $x, y \in S$ such that $d(x, y)=2$. Let $z \in S \cap N_{G}(x) \cap_{G}(y)$. Then $S \backslash\{z\}$ and $(S \backslash\{z\}) \cup\{v\}$ are not convex sets for all $v \in(V(G) \backslash S) \cap N_{G}(z)$. This implies that $S$ is not a 1-movable convex dominating set of $G$, contrary to our assumption. Thus, $S$ is a clique dominating set of $G$. Next, let $u \in S$. Since $S$ is a 1-movable convex dominating set of $G$, either $S \backslash\{v\}$ is a convex dominating set or there exists a vertex $w \in(V(G) \backslash S) \cap N_{G}(u)$ such that $S_{u}=(S \backslash\{u\}) \cup\{w\}$ is a convex dominating set of $G$. If $S \backslash\{u\}$ is a convex dominating set, then it $S \backslash\{v\}$ is a clique dominating set since $\langle S \backslash\{u\}\rangle$ is a clique (because $\langle S\rangle$ is a clique). Suppose $(S \backslash\{v\}) \cup\{w\}$ is a convex dominating set of $G$ for some $w \in(V(G) \backslash S) \cap N_{G}(u)$. Suppose further that there exists $q \in(S \backslash\{u\}) \backslash N_{G}(w)$. Then $[q, u, w]$ is a $q-w$ geodesic. Hence, $(S \backslash\{v\}) \cup\{w\}$ is not a convex set, contrary to our assumption. Therefore $S \backslash\{v\}) \cup\{w\}$ is a clique dominating set of $G$. Accordingly, $S$ is a 1-movable clique dominating set of $G$.

The converse follows from the fact that every subset of $V(G)$ that induces a clique is a convex set.

The next result is a direct consequence of Theorem 2.1.

Corollary 2.2. Let $G$ be a connected graph. If $G$ has a 1-movable clique dominating set, then $\gamma_{m c l}^{1}(G)=\gamma_{m c o n}^{1}(G)$.

Remark 2.3. Let $G$ be a connected graph. Then $\gamma_{c l}(G)=1$ if and only if $\gamma(G)=1$.

Theorem 2.4. Let $G$ be a connected nontrivial graph. Then the following are equivalent:

(i) $\gamma_{m}^{1}(G)=1$ 
(ii) $\gamma_{m c l}^{1}(G)=1$

(iii) $G=K_{2}$ or $G=K_{2}+H$ for some graph $H$.

Proof. $(i) \Longrightarrow(i i)$. Assume that $\gamma_{m}^{1}(G)=1$ and $S$ be a $\gamma_{m}^{1}$-set of $G$. Since $\langle S\rangle=K_{1}, S$ is a 1-movable clique dominating set of $G$. Thus, $\gamma_{m c l}^{1}(G)=1$.

$(i i) \Longrightarrow($ iii $)$. Suppose that $\gamma_{m c l}^{1}(G)=1$, say $S=\{x\}$ is a $\gamma_{m c l}^{1}$-set of $G$. Since $S$ is a 1-movable clique dominating set of $G$, there exists $y \in(V(G) \backslash$ $\{x\}) \cap N_{G}(x)$ such that $(S \backslash\{x\}) \cup\{y\}$ is a dominating set of $G$. Let $H=$ $\langle V(G) \backslash\{x, y\}\rangle$. Then $G=\langle\{x, y\}\rangle+H \cong K_{2}+H$.

$($ iii $) \Longrightarrow(i)$. Suppose first that $G=K_{2}$. Then $\gamma_{m c l}^{1}(G)=1$. This implies that $\gamma_{m}^{1}(G)=1$. Suppose that $G \cong K_{2}+H$. Let $V\left(K_{2}\right)=\{a, b\}$ and let $S=$ $\{a\}$. Then $S$ is as 1-movable dominating set of $G$. Therefore, $\gamma_{m}^{1}(G)=1$.

Theorem 2.5. Let $G$ be a connected graph of order $n \geq 3$. Then $\gamma_{m c l}^{1}(G)=2$ if and only if there exist adjacent vertices $x$ and $y$ of $G$ satisfying the following properties:

(i) $N_{G}(x) \cup N_{G}(y)=V(G)$

(ii) $N_{G}[x] \backslash N_{G}[y] \neq \varnothing$ or $N_{G}[y] \backslash N_{G}[x] \neq \varnothing$

(iii) $\{x\}$ is a dominating set of $G$ or there exists $v \in(V(G) \backslash S) \cap N_{G}(y)$ such that $N_{G}[y] \backslash N_{G}(x) \subseteq N_{G}(v)$

(iv) $\{y\}$ is a dominating set of $G$ or there exists $w \in(V(G) \backslash S) \cap N_{G}(x)$ such that $N_{G}[x] \backslash N_{G}(y) \subseteq N_{G}(w)$.

Proof. Let $G$ be a connected graph of order $n \geq 3$ such that $\gamma_{m c l}^{1}(G)=$ 2. Let $S=\{x, y\}$ be a $\gamma_{m c l}^{1}$ set of $G$. Then $x$ and $y$ are adjacent vertices. Clearly, $N_{G}(x) \cup N_{G}(y) \subseteq V(G)$. Let $v \in V(G)$. Since $S$ is a 1-movable clique dominating set of $G$, either $v x \in E(G)$ or $v y \in E(G)$. This means that either $v \in N_{G}(x)$ or $v \in N_{G}(y)$, that is, $v \in N_{G}(x) \cup N_{G}(y)$. It follows that $V(G) \subseteq$ $N_{G}(x) \cup N_{G}(y)$, and $(i)$ is proved. Next, suppose that $N_{G}[x] \backslash N_{G}[y]=\varnothing$ and $N_{G}[y] \backslash N_{G}[x]=\varnothing$. This implies that $N_{G}[x]=N_{G}[y]$ and so there is some graph $H$ such that $G \cong K_{2}+H$. By Theorem 2.4, $\gamma_{m c l}^{1}(G)=1$, a contradiction to the hypothesis. Therefore, either $N_{G}[x] \backslash N_{G}[y] \neq \varnothing$ or $N_{G}[y] \backslash N_{G}[x] \neq \varnothing$. This proves $(i i)$. Since $S$ is a 1-movable clique dominating set of $G$, either $S \backslash\{y\}=\{x\}$ is a dominating set of $G$ or there exists $v \in(V(G) \backslash S) \cap N_{G}(y)$ such that $(S \backslash\{y\}) \cup\{v\}=\{x, v\}$ is a clique dominating set of $G$. Let $a \in$ $N_{G}[y] \backslash N_{G}(x)$. Then $a \in N_{G}[y]$ but $a \notin N_{G}(x)$. Thus, $a$ is a neighbor of $v$, that is, $a \in N_{G}(v)$. Hence, $N_{G}[y] \backslash N_{G}(x) \subseteq N_{G}(v)$. This proves (iii). Similarly, (iv) holds. 
For the converse, suppose that there exist $x, y \in V(G)$ such that $x$ and $y$ are adjacent and satisfy conditions $(i)$ to $(i v)$. We claim that $S=\{x, y\}$ is a 1-movable clique dominating set of $G$. Clearly, $\langle S\rangle=K_{2}$, and so is complete. Let $a \in V(G) \backslash S$. Then by $(i), a \in\left(N_{G}(x) \cup N_{G}(y)\right) \backslash\{x, y\}$. This means that $a \neq x, a \neq y$ and $a x \in E(G)$ or $a y \in E(G)$. Since $a$ is arbitrary, $S$ is a dominating set of $G$. Consequently, $S$ is a clique dominating set of $G$. Now consider $\{x\}$. If $\{x\}$ is a dominating set of $G$, then $\gamma(G)=1$. By Theorem 2.4, $\gamma_{c l}(G)=1$, that is, $\{x\}$ is a clique dominating set of $G$. Suppose not. Then there exists $v \in(V(G) \backslash\{x, y\}) \cap N_{G}(y)$ such that $N_{G}[y] \backslash N_{G}(x) \subseteq N_{G}(v)$. Note that $x \in N_{G}[y] \backslash N_{G}(x) \subseteq N_{G}(v)$. This shows that $x$ is a neighbor of $v$. It follows that $\langle\{x, v\}\rangle=K_{2}$. Now, let $p \in V(G) \backslash\{x, y\}$. Then $p \in\left(N_{G}(x) \cup N_{G}(y)\right) \backslash\{x, y\}$ implying that $p \in N_{G}(x)$ or $p \in N_{G}(y)$. If $p \in N_{G}(x)$, then we are done. Suppose that $p \notin N_{G}(x)$. Then $p \in N_{G}(y)$. Thus, $p \in N_{G}(y) \backslash N_{G}(x) \subseteq$ $N_{G}[y] \backslash N_{G}(x) \subseteq N_{G}(v)$. Hence, $p v \in E(G)$. Consequently, $\{x, v\}$ is a clique dominating set of $G$. Similarly, if we consider $y$, then either $\{y\}$ is a clique dominating set of $G$ or there exists $w \in(V(G) \backslash S) \cap N_{G}(x)$ such that $\{y, w\}$ is a clique dominating set of $G$. This shows that $S$ is a 1-movable clique dominating set of $G$. Suppose $\gamma_{m c l}^{1}(G)=1$. Then either $G \cong K_{2}$ or $G \cong K_{2}+H$ for some graph $H$. Since $n \geq 3$ and $\langle S\rangle=K_{2}, G=S+H$. Thus, $N_{G}[x]=N_{G}[y]$. Hence, $N_{G}[x] \backslash N_{G}[y]=\varnothing$ and $N_{G}[y] \backslash N_{G}[x]=\varnothing$, a contradiction. Since $S$ is a 1-movable clique dominating set of $G$ and $|S|=2, \gamma_{m c l}^{1}(G)=2$.

Theorem 2.6. Let $G$ and $H$ be any two nonempty graphs. A subset $S$ of $V(G+H)$ is a 1-movable clique dominating set of $G+H$ if and only if one of the following statements holds:

(i) $S$ is a clique dominating set of $G$ such that if $|S|=1$, then either $S$ is a 1-movable dominating set of $G$ or there exists $b \in V(H)$ such that $\{b\}$ is a dominating set of $H$.

(ii) $S$ is a clique dominating set of $H$ such that if $|S|=1$, then either $S$ is a 1-movable dominating set of $H$ or there exists $a \in V(G)$ such that $\{a\}$ is a dominating set of $G$.

(iii) $S=\{a, b\}$, where one of the following is satisfied:

(1) $a$ and $b$ are not isolated vertices of $G$ and $H$, respectively.

(2) $\{a\}$ is a dominating set of $G$.

(3) $\{a, v\}$ is a clique dominating set of $G$ for some $v \in V(G)$.

(4) $\{b\}$ is a dominating set of $H$. 
(5) $\{b, w\}$ is a clique dominating set of $H$ for some $w \in V(H)$.

(iv) $S=\{a\} \cup E$, where $|E| \geq 2$, $a$ is not an isolated vertex of $G$ and $\langle E\rangle$ is a clique in $H$, or $E$ is a clique dominating set of $H$, or $E \cup\{v\}$ is a clique dominating set of $H$ for some $v \in V(H) \backslash E$.

(v) $S=D \cup\{b\}$, where $|D| \geq 2, b$ is not an isolated vertex of $H$ and $\langle D\rangle$ is a clique in $G$, or $D$ is a clique dominating set of $G$, or $D \cup\{w\}$ is a clique dominating set of $G$ for some $w \in V(G) \backslash D$.

(vi) $\left|S_{1}\right| \geq 2$ and $\left|S_{2}\right| \geq 2$, where $\left\langle S_{1}\right\rangle$ and $\left\langle S_{2}\right\rangle$ are cliques in $G$ and $H$, respectively.

Proof. Suppose $S$ is a 1 -movable clique dominating set of $G+H$. Consider the following cases:

Case 1. $S \cap V(H)=\varnothing$ or $S \cap V(G)=\varnothing$

Suppose that $S \cap V(H)=\varnothing$. Then $S \subseteq V(G)$ and is a clique dominating set of $G$. Suppose that $|S|=1$, say $S=\{x\}$ for some $x \in V(G)$. Since $S$ is a 1-movable clique dominating set of $G+H$, there exists $b \in V(G+H)$ such that $(S \backslash\{x\}) \cup\{b\}=\{b\}$ is a clique dominating set of $G+H$. If $b \in V(G)$, then $\{b\}$ is a dominating set of $G$. Hence, $S$ is a 1-movable clique dominating set of $G$. If $b \in V(H)$, then $\{b\}$ is a dominating set of $H$. Thus, $(i)$ holds. Similarly, (ii) holds if $S \cap V(G)=\varnothing$.

Case 2. $S_{1}=S \cap V(G) \neq \varnothing$ and $S_{2}=S \cap V(H) \neq \varnothing$

Consider the following subcases.

Subcase 1. $\left|S_{1}\right|=\left|S_{2}\right|=1$

Since $S$ is a 1-movable clique dominating set of $G+H, a$ and $b$ cannot be both isolated vertices. If $a$ and $b$ are both non-isolated vertices, then (1) of $(i i i)$ holds. Suppose that $a$ or $b$ is an isolated vertex, say $b$ is an isolated vertex of $H$. Since $S$ is a 1-movable clique dominating set of $G+H, S \backslash\{b\}=\{a\}$ is a dominating set of $G+H$ or there exists $v \in(V(G+H) \backslash S) \cap N_{G+H}(b)$ such that $(S \backslash\{b\}) \cup\{v\}=\{a, v\}$ is a clique dominating set of $G+H$. Since $b$ is an isolated vertex, $\{a, v\}$ is a clique dominating set of $G$. Hence, (2) or (3) of (iii) holds. Similarly, (4) or (5) of (iii) holds if $a$ is an isolated vertex.

Subcase 2. $\left|S_{1}\right|=1$ and $\left|S_{2}\right| \geq 2$ or $\left|S_{1}\right| \geq 2$ and $\left|S_{2}\right|=1$

Let $S_{1}=\{a\}$. Since $\langle S\rangle$ is a clique, $\left\langle S_{2}\right\rangle$ is a clique in $H$. If $a$ is not an isolated vertex of $G$, then we are done. Suppose $a$ is an isolated vertex of $G$. Since $S$ is a 1-movable clique dominating set $G+H$, either $S \backslash\{a\}=$ $S_{2}$ is a clique dominating set of $H$ or there exists $v \in V(H) \backslash S_{2}$ such that $(S \backslash\{a\}) \cup\{v\}=S_{2} \cup\{v\}$ is a clique dominating set of $H$. Hence, $(i v)$ holds. Similarly, $(v)$ holds if $\left|S_{1}\right| \geq 2$ and $\left|S_{2}\right|=1$. 
Subcase 3. $\left|S_{1}\right| \geq 2$ and $\left|S_{2}\right| \geq 2$

Since $\langle S\rangle$ is a clique and $S=S_{1} \cup S_{2}$, it follows that $\left\langle S_{1}\right\rangle$ and $\left\langle S_{2}\right\rangle$ are cliques in $G$ and $H$, respectively.

The converse is easy.

The next result is an immediate consequence of Theorem 2.6.

Corollary 2.7. Let $G$ and $H$ be nonempty graphs. Then $1 \leq \gamma_{m c l}^{1}(G+$ $H) \leq 2$. Moreover, $\gamma_{m c l}^{1}(G+H)=1$ if and only if one of the following statements holds:

(1) $\gamma_{m c l}^{1}(G)=1$

(2) $\gamma(G)=1$ and $\gamma(H)=1$

(3) $\gamma_{m c l}^{1}(H)=1$

We now characterize the 1-movable clique dominating sets in the composition of graphs.

Theorem 2.8. Let $G$ and $H$ be connected nontrivial graphs such that $G$ has a clique dominating set. A subset $C=\bigcup_{x \in S}\left[\{x\} \times T_{x}\right]$, where $S \subseteq V(G)$ and $T_{x} \subseteq V(H)$ for each $x \in S$, is a 1-movable clique dominating set of $G[H]$ if and only if $S$ is a clique dominating set of $G$ such that

(i) $\left\langle T_{x}\right\rangle$ is a clique in $H$ for each $x \in S$

(ii) $T_{x}$ is a clique dominating set of $H$ whenever $S=\{x\}$ and $\left|T_{x}\right| \geq 2$.

(iii) $T_{x}$ is a 1-movable clique dominating set of $H$ or $T_{x}$ is a clique dominating set of $H$ and $S$ is a 1-movable clique dominating set of $G$ whenever $S=$ $\{x\}$ and $\left|T_{x}\right|=1$.

Proof. Suppose that $C$ is a 1 -movable clique dominating set of $G[H]$. Then $C$ is a clique dominating set of $G[H]$. Hence, $S$ is a clique dominating set of $G$. Let $x \in S$ and let $a, b \in T_{x}$, where $a \neq b$. Since $\langle C\rangle$ is a clique in $G[H]$, $(x, a)(x, b) \in E(G[H])$. It follows that $a b \in E(H)$, showing that $\left\langle T_{x}\right\rangle$ is a clique in $H$. Next, suppose that $S=\{x\}$. Let $c \in V(H) \backslash T_{x}$. Since $C$ is a dominating set and $(x, c) \notin C$, it follows that there exists $(x, d) \in C \cap N_{G[H]}((x, c))$. This implies that $d \in T_{x} \cap N_{H}(c)$. Therefore, $T_{x}$ is a (clique) dominating set of $H$. Hence, $(i i)$ holds if $\left|T_{x}\right| \geq 2$. Suppose $\left|T_{x}\right|=1$. Let $a \in T_{x}$. Then $\{(x, a)\}=C$. Since $C$ is a 1 -movable clique dominating set of $G[H]$, there exists

$$
(y, b) \in(V(G[H]) \backslash C) \cap N_{G[H]}((x, a))
$$


such that $(C \backslash\{(x, a)\}) \cup\{(y, b)\}$ is a clique dominating set of $G[H]$. It follows that $T_{x}$ is a clique dominating set of $H$. If $x=y$, then $b \in\left(V(H) \backslash T_{x}\right) \cap N_{H}(a)$. This means that $\left(T_{x} \backslash\{a\}\right) \cup\{b\}=\{b\}$ is a clique dominating set of $H$. Thus, $T_{x}$ is a 1-movable clique dominating set of $H$. If $x \neq y$, then $y \in(V(G) \backslash S) \cap$ $N_{G}(x)$. Hence, $(S \backslash\{x\}) \cup\{y\}=\{y\}$ is a clique dominating set of $G$. Thus, $S$ is a 1-movable clique dominating set of $G$ showing that (iii) holds.

For the converse, suppose that $S$ is a clique dominating set of $G$ and $(i),(i i)$ and (iii) hold. Then, clearly, $C=\bigcup_{x \in S}\left[\{x\} \times T_{x}\right]$ induces a clique in $G[H]$. Let $(z, d) \notin C$. If $z \notin S$, then there exists $w \in S$ such that $w z \in E(G)$. Choose any $q \in T_{w}$. Then $(w, q) \in C \cap N_{G[H]}((z, d))$. Suppose $z \in S$. If $|S| \geq 2$, then there exists $y \in S \cap N_{G}(z)$. Pick any $p \in T_{y}$. Then $(y, p) \in C \cap N_{G[H]}((z, d))$. Suppose $S=\{z\}$. Then, by assumption, $T_{z}$ is a dominating set in $H$. Hence, there exists $t \in T_{z} \cap N_{H}(d)$. This implies that $(z, t) \in C \cap N_{G[H]}((z, d))$. Therefore, $C$ is a (clique) dominating set of $G[H]$.

Let $(x, a) \in C$. Consider the following cases:

Case 1. $S=\{x\}$

If $\left|T_{x}\right|=1$, that is, $T_{x}=\{a\}$, then $T_{x}$ is a 1-movable clique dominating set of $H$ or $T_{x}$ is a clique dominating set of $H$ and $S$ is a 1-movable clique dominating set of $G$. Hence, $C$ is a 1-movable clique dominating set of $G[H]$. Suppose $\left|T_{x}\right| \geq$ 2. Then there exists $y \in(V(G) \backslash\{x\}) \cap N_{G}(x)$. Thus, $(C \backslash\{(x, a)\}) \cup\{(y, b)\}$ is a clique dominating set of $G[H]$. Hence, $C$ is a 1-movable clique dominating set of $G[H]$.

Case 2. $|S| \geq 2$

If $T_{x}=\{a\}$, then there exists $b \in\left(V(H) \backslash T_{x}\right) \cap N_{H}(a)$. This means that $(C \backslash\{(x, a)\}) \cup\{(x, b)\}$ is a clique dominating set of $G[H]$. If $\left|T_{x}\right| \geq 2$, then $C \backslash\{(x, a)\}$ is a clique dominating set of $G[H]$. Therefore, $C$ is a 1-movable clique dominating set of $G[H]$.

Corollary 2.9. Let $G$ and $H$ be connected nontrivial graphs such that $G$ has a clique dominating set. Then

$$
\gamma_{m c l}^{1}(G[H])= \begin{cases}1, & \text { if } \gamma(G)=1 \text { and } \gamma_{m c l}^{1}(H)=1 \\ & \text { or } \gamma_{m c l}^{1}(G)=1 \text { and } \gamma(H)=1 \\ 2, & \text { if } \gamma(G)=1 \text { and } \gamma(H) \neq 1 \\ \gamma_{c l}(G), & \text { if } \gamma(G) \neq 1 .\end{cases}
$$




\section{Acknowledgments}

This research is funded by the DOST-ASTHRDP-NSC-SRSF, Philippines. The authors also acknowledge the reviewers for the comments and suggestions they made which contributed much for the improvement of the paper.

\section{References}

[1] J. Blair, R. Gera, S. Horton, Movable dominating sensor sets in networks, Networks, $7 \mathbf{7}$ (2011), 103-123.

[2] S.R. Canoy, Jr., I.J. Garces, Convex sets under some graph operations. Graphs and Combinatorics, 18 (2002), 787-793.

[3] G. Chartrand, P. Zhang, Convex sets in graphs, Congressus Numerantium, 136 (1999), 19-32.

[4] M. B. Cozzens, L. Kelleher, Dominating cliques in graphs, Journal of Combinatorial Mathematics and Combinatorial Computing, 86 (1990), 101-116.

[5] F. Harary, J. Nieminen, Convexity in graphs, J. Differential Geom., 16 (1981), 185-190.

[6] T.W. Haynes, S.T. Hedetniemi, P.J. Slater, Fundamentals of domination in graphs. Marcel Dekker, New York (1998).

[7] R. Hinampas, Jr., S.R. Canoy, Jr., 1-movable domination in graphs, Applied Mathematical Sciences, 8 (2014), 8565-8571.

[8] M.A. Labendia, S.R. Canoy, Jr., Convex domination in the composition and Cartesian product of graphs, Czechoslovak Mathematical Journal, 62 (2012), 1003-1009.

[9] M. Lemanska, Weakly convex and convex domination numbers, Opuscula Mathematica, 24 (2004), 181-188. 
